\title{
Sharp bounds for the Jensen divergence with applications
}

\author{
S. S. Dragomir, N. M. Dragomir, and D. Sherwell
}




\title{
SHARP BOUNDS FOR THE JENSEN DIVERGENCE WITH APPLICATIONS
}

\section{S. S. DRAGOMIR, N. M. DRAGOMIR, AND D. SHERWELL}

Received 24 September, 2013

\begin{abstract}
In this paper we provide sharp bounds for the Jensen divergence generated by different classes of functions including functions of bounded variation, absolutely continuous, Lipschitz continuous, convex functions and differentiable functions whose derivatives enjoy various properties as mentioned above. The bounds are expressed in terms of known and simpler divergence measures that are of importance in various applications such as the analysis of diversity as between and within populations and to cluster analysis.
\end{abstract}

2010 Mathematics Subject Classification: 26D15; 94017

Keywords: divergence measures, Jensen divergence, inequalities for real numbers

\section{INTRODUCTION}

For a function $\Phi$ defined on an interval $I$ of the real line $\mathbb{R}$, following Burbea and Rao [2], we consider the Jensen divergence (or simply f-divergence) between $x$ and $y$ from $I^{n}$ (where $n \geq 1$ ) defined by the quantity

$$
\mathcal{A}_{n, \Phi}(x, y):=\sum_{i=1}^{n}\left\{\frac{1}{2}\left[\Phi\left(x_{i}\right)+\Phi\left(y_{i}\right)\right]-\Phi\left(\frac{x_{i}+y_{i}}{2}\right)\right\},(x, y) \in I^{n} \times I^{n} \text {. }
$$

For a probability distribution $p_{1}, \ldots, p_{k} \geq 0(k \geq 2)$ with $\sum_{j=1}^{k} p_{j}=1$ the authors of [2] also considered the generalized mutual information measure defined by

$$
\mathcal{g}_{n, \Phi}^{p}\left(y^{1}, \ldots, y^{k}\right):=\sum_{i=1}^{n}\left\{\sum_{j=1}^{k} p_{j} \Phi\left(y_{i}^{j}\right)-\Phi\left(\sum_{j=1}^{k} p_{j} y_{i}^{j}\right)\right\}
$$

where $\left(y^{1}, \ldots, y^{k}\right) \in I^{n} \times \ldots \times I^{n}$.

For the convex function $\Phi:[0, \infty) \rightarrow \mathbb{R}, \Phi(t):=t \log t$ with the usual convention that $0 \log 0=0, \mathcal{F}_{n, \Phi}^{p}$ which is nonnegative, is the same as the mutual information (trans-information) $\mathcal{I}_{n}^{p}$ defined in information theory as a measure of information on a $k$-input channel for input distribution $p=\left(p_{1}, \ldots, p_{k}\right)$. 
For a discussion on the properties of $\mathcal{g}_{n}^{p}$ see Gallager [3, p. 16] and Aczél and Daróczy [1, 196-199].

In biological work, $g_{n}^{p}$ is defined to be the information radius on the probability distributions associated with $y^{1}, \ldots, y^{k}$ (see [12]). Applications of this concept to cluster analysis are discussed in [12] and [5]. For applications of $g_{n}^{p}$ in the analysis of diversity as between and within populations, see [6] and [10].

Define

$$
S_{n}:=\left\{\left(x_{1}, \ldots, x_{n}\right) \in I_{0}^{n}, \sum_{i=1}^{n} x_{i}=1\right\}, I_{0}:=(0,1) .
$$

We denote by

$$
\bar{S}_{n}:=\left\{\left(x_{1}, \ldots, x_{n}\right) \in \bar{I}_{0}^{n}, \sum_{i=1}^{n} x_{i}=1\right\}, \bar{I}_{0}:=[0,1]
$$

the closure of $S_{n}$.

Utilizing the family of functions

$$
\Phi_{\alpha}(t):= \begin{cases}(\alpha-1)^{-1}\left(t^{\alpha}-t\right), & \alpha \neq 1, \\ t \log t & \alpha=1,\end{cases}
$$

by Havrda and Charvát in [4] to introduce their entropies of degree $\alpha$ we mention, as examples, the following family of Jensen divergences considered in [2]

$$
\mathcal{F}_{n, \alpha}(x, y):= \begin{cases}(\alpha-1)^{-1} \sum_{i=1}^{n}\left[\frac{1}{2}\left(x_{i}^{\alpha}+y_{i}^{\alpha}\right)-\left(\frac{x_{i}+y_{i}}{2}\right)^{\alpha}\right], & \alpha \neq 1 \\ \log \left[\frac{\prod_{i=1}^{n}\left(x_{i}^{x_{i}} y_{i}^{y_{i}}\right)^{1 / 2}}{\prod_{i=1}^{n}\left(\frac{x_{i}+y_{i}}{2}\right)^{\frac{x_{i}+y_{i}}{2}}}\right], & \alpha=1\end{cases}
$$

that can be extended on $\bar{S}_{n} \times \bar{S}_{n}$ with the usual convention that $0 \log 0=0$.

The divergence $\mathcal{J}_{n, 1}$, written in its equivalent form as

$$
\mathcal{I}_{n, 1}(x, y):=\frac{1}{2} \sum_{i=1}^{n}\left\{x_{i} \log x_{i}+y_{i} \log y_{i}-\left(x_{i}+y_{i}\right) \log \left(\frac{x_{i}+y_{i}}{2}\right)\right\},
$$

is also known in the literature as the Jensen-Shannon divergence. Its important applications in various fields of Mathematics and Statistics can be found, for instance in $[8,9,11,13]$ and the references therein.

The convexity of divergence measures is an attractive feature. The following result concerning this property holds: 
Theorem 1 (Burbea-Rao, 1982, [2]). Let $\Phi$ be a $C^{2}$ function on the interval of real numbers $I$. Then $\mathcal{I}_{n, \Phi}$ is convex (concave) on $I^{n} \times I^{n}$ if and only if $\Phi$ is convex (concave) and $\frac{1}{\Phi^{\prime \prime}}$ is concave (convex) on I. Further, in this case $\mathcal{G}_{n, \Phi}^{p}$ is also convex (concave) on $I^{n k}$ for any given probability distribution $p$.

In this paper we endeavour to provide sharp upper and lower bounds for the Jensen divergence for various classes of functions $\Phi$, including functions of bounded variation, absolutely continuous functions, Lipschitzian continuous functions, convex functions and differentiable functions whose derivative belong to the above mentioned classes. The bounds will be expressed in terms of known and simpler divergence measures that have been employed in various applications as mentioned above.

\section{Some General Results}

The following result may be stated

Proposition 1. If $\Phi:[a, b] \rightarrow \mathbb{R}$ is a bounded function with $-\infty<m \leq \Phi(t) \leq$ $M<\infty$ for any $t \in[a, b]$, then

$$
\left|\mathcal{g}_{n, \Phi}(x, y)\right| \leq n(M-m) .
$$

For a fixed $n$, the multiplicative constant 1 in front of $M-m$ cannot be replaced by a smaller quantity.

Proof. For the sake of completeness, we present a short proof.

Since $\Phi$ is bounded, we have $m \leq \Phi(x) \leq M, m \leq \Phi(y) \leq M$ and $-M \leq$ $-\Phi\left(\frac{x+y}{2}\right) \leq-m$, which gives, by addition that

$$
-(M-m) \leq \frac{\Phi(x)+\Phi(y)}{2}-\Phi\left(\frac{x+y}{2}\right) \leq M-m,
$$

for each $x, y \in[a, b]$, i.e.,

$$
\left|\frac{\Phi(x)+\Phi(y)}{2}-\Phi\left(\frac{x+y}{2}\right)\right| \leq M-m
$$

which implies the desired inequality (2.1).

Let us prove the sharpness of the constant for the case $n=1$.

If $\Phi:[a, b] \rightarrow \mathbb{R}, \Phi(t)=\left|t-\frac{a+b}{2}\right|$, then $\Phi(a)=\Phi(b)=\frac{b-a}{2}, \Phi\left(\frac{a+b}{2}\right)=0$, $M=\frac{b-a}{2}$ and $m=0$ and the inequality (2.2) becomes an equality with both terms equal to $\frac{b-a}{2}$.

Proposition 2. Let $\Phi:[a, b] \rightarrow \mathbb{R}$ be a function of bounded variation on the compact interval $[a, b]$ of real numbers $\mathbb{R}$. Then for any $x=\left(x_{1}, \ldots, x_{n}\right), y=$ 
$\left(y_{1}, \ldots, y_{n}\right) \in[a, b]^{n}$ we have the inequality

$$
\left|\mathcal{I}_{n, \Phi}(x, y)\right| \leq \frac{1}{2} \sum_{i=1}^{n}\left|\bigvee_{x_{i}}^{y_{i}}(\Phi)\right| \leq \frac{1}{2} n \bigvee_{a}^{b}(\Phi)
$$

where $\bigvee_{a}^{b}(\Phi)$ denotes the total variation of $\Phi$ on $[a, b]$.

The constant $\frac{1}{2}$ is best possible in both inequalities from (2.3).

Proof. It suffices to prove the inequality for $n=1$.

Assume that $x<y$. Then

$$
\begin{aligned}
& \left|\frac{\Phi(x)+\Phi(y)}{2}-\Phi\left(\frac{x+y}{2}\right)\right| \\
& \leq \frac{1}{2}\left[\left|\Phi\left(\frac{x+y}{2}\right)-\Phi(x)\right|+\left|\Phi(y)-\Phi\left(\frac{x+y}{2}\right)\right|\right] \leq \frac{1}{2} \bigvee_{x}^{y}(\Phi) .
\end{aligned}
$$

By symmetry reasons, we deduce a similar inequality when $y<x$. Therefore, for any $x, y \in I$ we have

$$
\left|\frac{\Phi(x)+\Phi(y)}{2}-\Phi\left(\frac{x+y}{2}\right)\right| \leq \frac{1}{2}\left|\bigvee_{x}^{y}(\Phi)\right|
$$

which implies the desired inequality (2.3).

The last part of (2.3) is obvious since $\left|\bigvee_{x_{i}}^{y_{i}}(\Phi)\right| \leq \bigvee_{a}^{b}(\Phi)$ for any selection of vectors $x=\left(x_{1}, \ldots, x_{n}\right), y=\left(y_{1}, \ldots, y_{n}\right) \in[a, b]^{n}$.

Now, if we take the function $\Phi:[0,1] \rightarrow \mathbb{R}, \Phi(x)=\operatorname{sgn}\left(x-\frac{1}{2}\right)$, then this function is of bounded variation on $[0,1]$ and if we take $x=0$ and $x=1$ then we have $\bigvee_{0}^{1}(\Phi)=2$ and we get in both sides of (2.4) the same quantity 1 , which proves the sharpness of the constant $\frac{1}{2}$ in both inequalities (2.3).

Corollary 1. Let $\Phi:[a, b] \rightarrow \mathbb{R}$ be a L-Lipschitzian function on $[a, b]$, i.e. we recall that $\Phi$ satisfies the condition

$$
|\Phi(t)-\Phi(s)| \leq L|t-s| \text { for any } t, s \in[a, b]
$$

where $L>0$ is given.

Then for any $x=\left(x_{1}, \ldots, x_{n}\right), y=\left(y_{1}, \ldots, y_{n}\right) \in[a, b]^{n}$ we have the inequality

$$
\left|\mathcal{I}_{n, \Phi}(x, y)\right| \leq \frac{1}{2} L \sum_{i=1}^{n}\left|x_{i}-y_{i}\right|=L \delta(x, y),
$$

where $\delta(x, y):=\frac{1}{2} \sum_{i=1}^{n}\left|x_{i}-y_{i}\right|$ is known in the literature as the statistical distance between $x$ and $y$.

The constant $\frac{1}{2}$ is best possible in (2.5). 
Proof. It is well known that, any $L$-Lipschitzian function on $[a, b]$ is of bounded variation on $[a, b]$ and $\bigvee_{a}^{b}(\Phi) \leq L(b-a)$. Applying this property to the inequality (2.3), we deduce the desired result (2.5).

We prove the sharpness esof the constant $\frac{1}{2}$ for the case $n=1$, i.e.,

$$
\left|\frac{\Phi(x)+\Phi(y)}{2}-\Phi\left(\frac{x+y}{2}\right)\right| \leq \frac{1}{2} L|x-y|
$$

is a sharp inequality provided $\Phi:[a, b] \rightarrow \mathbb{R}$ is a $L$-Lipschitzian function on $[a, b]$.

If we consider the function $\Phi:[a, b] \rightarrow[0, \infty)$ defined by $\Phi(t)=\left|t-\frac{a+b}{2}\right|$, then $\Phi$ is Lipschitzian with the constant $L=1$ and if we use this function and $x=a, y=b$ in (2.6) we obtain the same quantity $\frac{1}{2}(b-a)$ in both sides.

This proves the desired result.

The following lemma may be stated.

Lemma 1. Let $u:[a, b] \rightarrow \mathbb{R}$ and $\gamma, \Gamma \in \mathbb{R}$ with $\Gamma>\gamma$. The following statements are equivalent:

(i) The function $u-\frac{\gamma+\Gamma}{2} \cdot e$, where $e(t)=t, t \in[a, b]$, is $\frac{1}{2}(\Gamma-\gamma)$-Lipschitzian;

(ii) We have the inequality:

$$
\gamma \leq \frac{u(t)-u(s)}{t-s} \leq \Gamma \quad \text { for each } t, s \in[a, b] \quad \text { with } t \neq s ;
$$

(iii) We have the inequality:

$$
\gamma(t-s) \leq u(t)-u(s) \leq \Gamma(t-s) \text { for each } t, s \in[a, b] \quad \text { with } t>s .
$$

Following [7], we can introduce the concept:

Definition 1. A function $u:[a, b] \rightarrow \mathbb{R}$ which satisfies one of the equivalent conditions (i) - (iii) is said to be $(\gamma, \Gamma)$-Lipschitzian on $[a, b]$.

Notice that in [6], the definition was introduced on utilizing the statement (iii) and only the equivalence (i) $\Leftrightarrow$ (iii) was considered.

Utilizing Lagrange's mean value theorem, we can state the following result that provides practical examples of $(\gamma, \Gamma)$-Lipschitzian functions.

Proposition 3. Let $u:[a, b] \rightarrow \mathbb{R}$ be continuous on $[a, b]$ and differentiable on $(a, b)$. If

$$
-\infty<\gamma:=\inf _{t \in(a, b)} u^{\prime}(t), \quad \sup _{t \in(a, b)} u^{\prime}(t)=: \Gamma<\infty
$$

then $u$ is $(\gamma, \Gamma)$-Lipschitzian on $[a, b]$.

We can improve the inequality (2.6) as follows: 
Corollary 2. Let $\Phi:[a, b] \rightarrow \mathbb{R}$ be $(\gamma, \Gamma)$-Lipschitzian on $[a, b]$ with $\gamma<\Gamma$. Then for any $x=\left(x_{1}, \ldots, x_{n}\right), y=\left(y_{1}, \ldots, y_{n}\right) \in[a, b]^{n}$ we have the inequality

$$
\left|\mathscr{g}_{n, \Phi}(x, y)\right| \leq \frac{1}{4}(\Gamma-\gamma) \sum_{i=1}^{n}\left|x_{i}-y_{i}\right|=\frac{1}{2}(\Gamma-\gamma) \delta(x, y) \text {. }
$$

The constant $\frac{1}{4}$ is best possible in (2.7).

In particular, if $\Phi:[a, b] \rightarrow \mathbb{R}$ is differentiable on $(a, b)$ and the derivative $\Phi^{\prime}$ satisfies the inequality $-\infty<\gamma \leq \Phi^{\prime}(t) \leq \Gamma<\infty$ for each $t \in(a, b)$, then (2.7) is valid as well.

Proof. Follows by Corollary 1 on taking into account that

$$
\mathcal{J}_{n, \Phi-\frac{\gamma+\Gamma}{2} \cdot e}(x, y)=\mathcal{A}_{n, \Phi}(x, y)
$$

and the details are omitted.

We recall that a function $f:[a, b] \rightarrow \mathbb{R}$ is absolutely continuous on $[a, b]$ if and only if it is differentiable almost everywhere in $[a, b]$. The derivative $f^{\prime}$ is Lebesgue integrable on this interval and $f(y)-f(x)=\int_{x}^{y} f^{\prime}(t) d t$ for any $x, y \in[a, b]$.

We use the following notations for Lebesgue integrable functions:

$$
\|g\|_{[x, y], p}:=\left.\left.\left|\int_{x}^{y}\right| g(s)\right|^{p} d s\right|^{1 / p} \text { if } 1 \leq p<\infty, x, y \in[a, b] \text { and } g \in L_{p}[a, b] \text {; }
$$

and for $g \in L_{\infty}[a, b]$ we denote

$$
\|g\|_{[x, y], \infty}:= \begin{cases}\operatorname{ess} \sup _{s \in[x, y]}|g(s)| & \text { if } x<y \\ \operatorname{esssup}_{s \in[y, x]}|g(s)| & \text { if } y<x .\end{cases}
$$

Theorem 2. Assume that $\Phi:[a, b] \rightarrow \mathbb{R}$ is absolutely continuous on $[a, b]$. Then we have the bounds

$$
\begin{aligned}
& \left|\mathscr{I}_{n, \Phi}(x, y)\right| \\
& \leq \frac{1}{2} \times \begin{cases}\sum_{i=1}^{n}\left|y_{i}-x_{i}\right|\left\|\Phi^{\prime}\right\|_{\left[x_{i}, y_{i}\right], \infty} & \text { if } \Phi^{\prime} \in L_{\infty}[a, b] \\
\sum_{i=1}^{n}\left|y_{i}-x_{i}\right|^{\frac{p-1}{p}}\left\|\Phi^{\prime}\right\|_{\left[x_{i}, y_{i}\right], p} & \text { if } \Phi^{\prime} \in L_{p}[a, b], p>1 \\
\sum_{i=1}^{n}\left\|\Phi^{\prime}\right\|_{\left[x_{i}, y_{i}\right], 1} & \end{cases} \\
& \leq \frac{1}{2} \times \begin{cases}\left\|\Phi^{\prime}\right\|_{[a, b], \infty} \sum_{i=1}^{n}\left|y_{i}-x_{i}\right| & \text { if } \Phi^{\prime} \in L_{\infty}[a, b] \\
\left\|\Phi^{\prime}\right\|_{[a, b], p} \sum_{i=1}^{n}\left|y_{i}-x_{i}\right|^{\frac{p-1}{p}} & \text { if } \Phi^{\prime} \in L_{p}[a, b], p>1 \\
n\left\|\Phi^{\prime}\right\|_{[a, b], 1} & \end{cases}
\end{aligned}
$$


for any $x=\left(x_{1}, \ldots, x_{n}\right), y=\left(y_{1}, \ldots, y_{n}\right) \in[a, b]^{n}$.

Moreover, if the modulus of the derivative is convex, then we have the inequality

$$
\begin{aligned}
\left|g_{n, \Phi}(x, y)\right| & \leq \frac{1}{4} \sum_{i=1}^{n}\left|y_{i}-x_{i}\right|\left[\left|\Phi^{\prime}\left(\frac{x_{i}+y_{i}}{2}\right)\right|+\frac{\left|\Phi^{\prime}\left(x_{i}\right)\right|+\left|\Phi^{\prime}\left(y_{i}\right)\right|}{2}\right] \\
& \leq \frac{1}{4} \sum_{i=1}^{n}\left|y_{i}-x_{i}\right|\left[\left|\Phi^{\prime}\left(x_{i}\right)\right|+\left|\Phi^{\prime}\left(y_{i}\right)\right|\right] \\
& \left(\leq\left\|\Phi^{\prime}\right\|_{[a, b], \infty} \delta(x, y)\right),
\end{aligned}
$$

for any $x=\left(x_{1}, \ldots, x_{n}\right), y=\left(y_{1}, \ldots, y_{n}\right) \in[a, b]^{n}$.

The constant $\frac{1}{4}$ is best possible in both inequalities.

Proof. Assume that $x<y$. Then we have

$$
\begin{aligned}
\left|\frac{\Phi(x)+\Phi(y)}{2}-\Phi\left(\frac{x+y}{2}\right)\right| & =\left|\frac{1}{2} \int_{x}^{\frac{x+y}{2}} \Phi^{\prime}(s) d s+\frac{1}{2} \int_{\frac{x+y}{2}}^{y} \Phi^{\prime}(s) d s\right| \\
& \leq \frac{1}{2}\left|\int_{x}^{\frac{x+y}{2}} \Phi^{\prime}(s) d s\right|+\frac{1}{2}\left|\int_{\frac{x+y}{2}}^{y} \Phi^{\prime}(s) d s\right| \\
& \leq \frac{1}{2} \int_{x}^{\frac{x+y}{2}}\left|\Phi^{\prime}(s)\right| d s+\frac{1}{2} \int_{\frac{x+y}{2}}^{y}\left|\Phi^{\prime}(s)\right| d s \\
& =\frac{1}{2} \int_{x}^{y}\left|\Phi^{\prime}(s)\right| d s .
\end{aligned}
$$

If $y<x$, then we also get

$$
\left|\frac{\Phi(x)+\Phi(y)}{2}-\Phi\left(\frac{x+y}{2}\right)\right| \leq \frac{1}{2} \int_{x}^{y}\left|\Phi^{\prime}(s)\right| d s .
$$

Therefore, for any $x, y \in[a, b]$ we have the inequality

$$
\left|\frac{\Phi(x)+\Phi(y)}{2}-\Phi\left(\frac{x+y}{2}\right)\right| \leq \frac{1}{2}\left|\int_{x}^{y}\right| \Phi^{\prime}(s)|d s| .
$$

Further, on making use of Hölder's integral inequality, we also have that

$$
\left|\int_{x}^{y}\right| \Phi^{\prime}(s)|d s| \leq \begin{cases}|y-x|\left\|\Phi^{\prime}\right\|_{[x, y], \infty} & \text { if } \Phi^{\prime} \in L_{\infty}[a, b] \\ |y-x|^{\frac{p-1}{p}}\left\|\Phi^{\prime}\right\|_{[x, y], p} & \text { if } \Phi^{\prime} \in L_{p}[a, b], p>1 .\end{cases}
$$

The above two inequalities (2.10) and (2.11) provide the result

$$
\left|\frac{\Phi(x)+\Phi(y)}{2}-\Phi\left(\frac{x+y}{2}\right)\right|
$$




$$
\leq \frac{1}{2} \times\left\{\begin{array}{cc}
|y-x|\left\|\Phi^{\prime}\right\|_{[x, y], \infty} & \text { if } \Phi^{\prime} \in L_{\infty}[a, b] \\
|y-x|^{\frac{p-1}{p}}\left\|\Phi^{\prime}\right\|_{[x, y], p} & \text { if } \Phi^{\prime} \in L_{p}[a, b], p>1 \\
\left\|\Phi^{\prime}\right\|_{[x, y], p} &
\end{array}\right.
$$

for any $x, y \in[a, b]$, which imply the first inequality in (2.8). The second inequality is obvious.

In order to prove the inequality (2.9) we use the following refinement of the celebrated Hermite-Hadamard inequality

$$
\begin{aligned}
\frac{1}{\beta-\alpha} \int_{\alpha}^{\beta} f(t) d t & \leq \frac{1}{2}\left[f\left(\frac{\alpha+\beta}{2}\right)+\frac{f(\alpha)+f(\beta)}{2}\right] \\
& \leq \frac{f(\alpha)+f(\beta)}{2}
\end{aligned}
$$

that works for a convex function $f:[a, b] \rightarrow \mathbb{R}$ and any $\alpha, \beta \in[a, b]$ with $\alpha \neq \beta$.

Applying this inequality for the convex function $\left|\Phi^{\prime}\right|$ we have

$$
\begin{aligned}
\left|\int_{x}^{y}\right| \Phi^{\prime}(s)|d s| & \leq \frac{1}{2}\left[\left|\Phi^{\prime}\left(\frac{x+y}{2}\right)\right|+\frac{\left|\Phi^{\prime}(x)\right|+\left|\Phi^{\prime}(y)\right|}{2}\right]|y-x| \\
& \leq \frac{1}{2}\left[\left|\Phi^{\prime}(x)\right|+\left|\Phi^{\prime}(y)\right|\right]|y-x|
\end{aligned}
$$

which together with (2.10) produces the required result (2.9).

Let us prove the sharpness of the constant $\frac{1}{4}$ for $n=1$, meaning that we need to prove that the inequalities

$$
\begin{aligned}
& \left|\frac{\Phi(x)+\Phi(y)}{2}-\Phi\left(\frac{x+y}{2}\right)\right| \\
& \leq \frac{1}{4}\left[\left|\Phi^{\prime}\left(\frac{x+y}{2}\right)\right|+\frac{\left|\Phi^{\prime}(x)\right|+\left|\Phi^{\prime}(y)\right|}{2}\right]|y-x| \\
& \leq \frac{1}{4}\left[\left|\Phi^{\prime}(x)\right|+\left|\Phi^{\prime}(y)\right|\right]|y-x|
\end{aligned}
$$

reduce to equality for some function $f$ and some numbers $x, y \in[a, b]$.

Consider $\Phi:[a, b] \rightarrow \mathbb{R}, \Phi(t)=\frac{1}{2}\left(t-\frac{a+b}{2}\right)^{2}$. Then $\Phi^{\prime}(t)=t-\frac{a+b}{2}$ and, obviously, $\left|\Phi^{\prime}\right|$ is a convex function.

If we replace this function in (2.14) and choose $x=a$ and $y=b$, then we get in all sides the same quantity $\frac{1}{4}(b-a)^{2}$.

\section{BOUndS For CONVEX Functions}

The case of convex functions is as follows: 
Theorem 3. Let $\Phi: I \rightarrow \mathbb{R}$ be a convex function on the interval I of real numbers $\mathbb{R}$. Then for any $x=\left(x_{1}, \ldots, x_{n}\right), y=\left(y_{1}, \ldots, y_{n}\right) \in \stackrel{\circ}{I}^{n}$, where $\stackrel{\circ}{I}$ denotes the interior of $I$, we have the inequalities

$$
\begin{aligned}
& \frac{1}{4} \sum_{i=1}^{n}\left(x_{i}-y_{i}\right)\left(\Phi_{+(-)}^{\prime}\left(x_{i}\right)-\Phi_{+(-)}^{\prime}\left(y_{i}\right)\right) \\
& \geq \mathcal{A}_{n, \Phi}(x, y) \\
& \geq \frac{1}{4} \sum_{i=1}^{n}\left|x_{i}-y_{i}\right|\left(\Phi_{+}^{\prime}\left(\frac{x_{i}+y_{i}}{2}\right)-\Phi_{-}^{\prime}\left(\frac{x_{i}+y_{i}}{2}\right)\right) \geq 0
\end{aligned}
$$

where $\Phi_{+(-)}^{\prime}(z)$ denote the right (left) derivative of $\Phi$ in $z$.

The constant $\frac{1}{4}$ is best possible in both inequalities.

Proof. It suffices to prove the inequality for $n=1$.

It is well know that, if $\Phi: I \rightarrow \mathbb{R}$ is a convex function on the interval $I$, then for any $x, y \in \stackrel{\circ}{I}$ we have the gradient inequality

$$
\mu(y)(y-x) \geq \Phi(y)-\Phi(x) \geq \lambda(x)(y-x)
$$

where $\mu(y) \in\left[\Phi_{-}^{\prime}(y), \Phi_{+}^{\prime}(y)\right]$ and $\lambda(x) \in\left[\Phi_{-}^{\prime}(x), \Phi_{+}^{\prime}(x)\right]$.

Now, making use of the second inequality in (3.2) and assuming that $x>y$ we can write that

$$
\Phi(x)-\Phi\left(\frac{x+y}{2}\right) \geq \frac{1}{2} \Phi_{+}^{\prime}\left(\frac{x+y}{2}\right)(x-y)
$$

and

$$
\Phi(y)-\Phi\left(\frac{x+y}{2}\right) \geq-\frac{1}{2} \Phi_{-}^{\prime}\left(\frac{x+y}{2}\right)(x-y) .
$$

If we multiply both inequalities with $\frac{1}{2}$ and add, we obtain

$$
\frac{1}{2}[\Phi(x)+\Phi(y)]-\Phi\left(\frac{x+y}{2}\right) \geq \frac{1}{4}(x-y)\left[\Phi_{+}^{\prime}\left(\frac{x+y}{2}\right)-\Phi_{-}^{\prime}\left(\frac{x+y}{2}\right)\right] .
$$

By symmetry reasons, if $y>x$ we also have

$$
\frac{1}{2}[\Phi(x)+\Phi(y)]-\Phi\left(\frac{x+y}{2}\right) \geq \frac{1}{4}(y-x)\left[\Phi_{+}^{\prime}\left(\frac{x+y}{2}\right)-\Phi_{-}^{\prime}\left(\frac{x+y}{2}\right)\right] .
$$

Therefore, for any $x, y \in \stackrel{\circ}{I}$ we have

$$
\begin{aligned}
\frac{1}{2}[\Phi(x)+\Phi(y)]-\Phi\left(\frac{x+y}{2}\right) & \geq \frac{1}{4}|y-x|\left[\Phi_{+}^{\prime}\left(\frac{x+y}{2}\right)-\Phi_{-}^{\prime}\left(\frac{x+y}{2}\right)\right] \\
& \geq 0,
\end{aligned}
$$

and the second inequality in (3.1) is proven. 
In order to prove the sharpness of the constant $\frac{1}{4}$ in (3.3), we consider the function $\Phi:[a, b] \rightarrow \mathbb{R}, \Phi(x)=\left|x-\frac{a+b}{2}\right|$. This function is convex and

$$
\Phi_{+}^{\prime}\left(\frac{a+b}{2}\right)=1 \text { while } \Phi_{-}^{\prime}\left(\frac{a+b}{2}\right)=-1 .
$$

If we write the inequality (3.3) for this convex function and $x=a, y=b$ we obtain in both sides of this inequality the same quantity $\frac{1}{2}(b-a)$ which proves the desired result.

Now, making use of the first inequality in (3.2) and assuming that $x, y \in \stackrel{\circ}{I}$ we can write that

$$
\frac{1}{2} \Phi_{+(-)}^{\prime}(x)(x-y) \geq \Phi(x)-\Phi\left(\frac{x+y}{2}\right)
$$

and

$$
-\frac{1}{2} \Phi_{+(-)}^{\prime}(y)(x-y) \geq \Phi(y)-\Phi\left(\frac{x+y}{2}\right) .
$$

If we multiply both inequalities with $\frac{1}{2}$ and add, we obtain

$$
\frac{1}{4}\left[\Phi_{+(-)}^{\prime}(x)-\Phi_{+(-)}^{\prime}(y)\right](x-y) \geq \frac{1}{2}[\Phi(x)+\Phi(y)]-\Phi\left(\frac{x+y}{2}\right)
$$

for any $x, y \in \stackrel{\circ}{I}$ and the first inequality in (3.1) is also proven.

If we consider the same function $\Phi:[a, b] \rightarrow \mathbb{R}, \Phi(x)=\left|x-\frac{a+b}{2}\right|$, then we observe that $\Phi_{-}^{\prime}(b)=1, \Phi_{+}^{\prime}(a)=-1$ and if we write the inequality (3.4) for this function and for $x=b, y=a$ we obtain in both sides of this inequality the same quantity $\frac{1}{2}(b-a)$.

Corollary 3. Let $\Phi: I \rightarrow \mathbb{R}$ be a differentiable convex function on the interval I of real numbers $\mathbb{R}$. Then for any $x=\left(x_{1}, \ldots, x_{n}\right), y=\left(y_{1}, \ldots, y_{n}\right) \in \stackrel{\circ}{I}^{n}$, we have the inequalities

$$
\frac{1}{4} \sum_{i=1}^{n}\left(x_{i}-y_{i}\right)\left(\Phi^{\prime}\left(x_{i}\right)-\Phi^{\prime}\left(y_{i}\right)\right) \geq \mathscr{J}_{n, \Phi}(x, y) \geq 0
$$

Remark 1. We observe that if $\Phi^{\prime}$ is $r-H$-Hölder continuous on $I$, i.e., there exist the constants $r \in(0,1]$ and $H>0$ such that $\left|\Phi^{\prime}(s)-\Phi^{\prime}(t)\right| \leq H|s-t|^{r}$ for any $s, t \in I$, then by (3.5) we have the upper bound

$$
\frac{1}{4} H \sum_{i=1}^{n}\left|x_{i}-y_{i}\right|^{r+1} \geq \mathscr{g}_{n, \Phi}(x, y) \geq 0
$$

and, in particular, for $r=1$, we have the bound

$$
H \operatorname{g}_{n, 2}(x, y) \geq \mathcal{A}_{n, \Phi}(x, y) \geq 0
$$


where, by (1.1),

$$
g_{n, 2}(x, y)=\sum_{i=1}^{n}\left[\frac{1}{2}\left(x_{i}^{2}+y_{i}^{2}\right)-\left(\frac{x_{i}+y_{i}}{2}\right)^{2}\right]=\frac{1}{4} \sum_{i=1}^{n}\left(x_{i}-y_{i}\right)^{2} .
$$

For two vectors $x=\left(x_{1}, \ldots, x_{n}\right), y=\left(y_{1}, \ldots, y_{n}\right) \in I^{n}$ we say that $x \leq y$ if for all $i \in\{1, \ldots, n\}$ we have that $x_{i} \leq y_{i}$. For $x \leq y$, we call the set $[x, y]:=$ $\left\{g=\left(g_{1}, \ldots, g_{n}\right) \mid\right.$ with $x_{i} \leq g_{i} \leq y_{i}$ for all $\left.i \in\{1, \ldots, n\}\right\}$ the generalized interval generated by $x$ and $y$.

Theorem 4. Let $\Phi: I \rightarrow \mathbb{R}$ be a convex function on the interval I of real numbers $\mathbb{R}$.

(i) If $x, y, z \in I^{n}$ are so that $x \leq y \leq z$, then

$$
0 \leq \mathscr{I}_{n, \Phi}(x, y)+\mathscr{I}_{n, \Phi}(y, z) \leq \mathscr{I}_{n, \Phi}(x, z),
$$

i.e., $\mathcal{I}_{n, \Phi}$ is superadditive as a functional of the generalized interval;

(ii) If $x, y, z, u \in I^{n}$ are so that $x \leq y \leq z \leq u$, then

$$
0 \leq \mathcal{I}_{n, \Phi}(y, z) \leq \mathcal{J}_{n, \Phi}(x, u),
$$

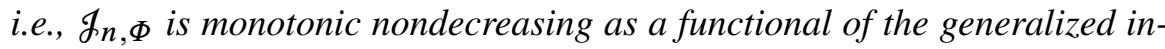
terval.

Proof. (i) It suffices to prove it for $n=1$.

So, assume that $x, y, z \in I$ with $x \leq y \leq z$. We claim that, if $\Phi: I \rightarrow \mathbb{R}$ is a convex function on the interval $I$, then

$$
\begin{aligned}
& \frac{1}{2}[\Phi(x)+\Phi(y)]-\Phi\left(\frac{x+y}{2}\right)+\frac{1}{2}[\Phi(y)+\Phi(z)]-\Phi\left(\frac{y+z}{2}\right) \\
& \leq \frac{1}{2}[\Phi(x)+\Phi(z)]-\Phi\left(\frac{x+z}{2}\right) .
\end{aligned}
$$

Observe that, this inequality actually reduces to

$$
\Phi(y)-\Phi\left(\frac{x+y}{2}\right) \leq \Phi\left(\frac{y+z}{2}\right)-\Phi\left(\frac{x+z}{2}\right) .
$$

If either $x=y$ or $y=z$, then (3.8) reduces to an equality, so we can suppose that $x<y<z$.

Now, for a convex function $\varphi: I \subset \mathbb{R} \rightarrow \mathbb{R}$, where $I$ is an interval, and any real numbers $t_{1}, t_{2}, s_{1}$ and $s_{2}$ from $I$ and with the properties that $t_{1} \leq s_{1}$ and $t_{2} \leq s_{2}$ we have that

$$
\frac{\varphi\left(t_{1}\right)-\varphi\left(t_{2}\right)}{t_{1}-t_{2}} \leq \frac{\varphi\left(s_{1}\right)-\varphi\left(s_{2}\right)}{s_{1}-s_{2}} .
$$

Indeed, since $\varphi$ is convex on $I$ then for any $a \in I$ the function $\psi: I \backslash\{a\} \rightarrow \mathbb{R}$

$$
\psi(t):=\frac{\varphi(t)-\varphi(a)}{t-a}
$$


is monotonic nondecreasing where it is defined. Utilizing this property repeatedly we have

$$
\begin{aligned}
\frac{\varphi\left(t_{1}\right)-\varphi\left(t_{2}\right)}{t_{1}-t_{2}} & \leq \frac{\varphi\left(s_{1}\right)-\varphi\left(t_{2}\right)}{s_{1}-t_{2}}=\frac{\varphi\left(t_{2}\right)-\varphi\left(s_{1}\right)}{t_{2}-s_{1}} \\
& \leq \frac{\varphi\left(s_{2}\right)-\varphi\left(s_{1}\right)}{s_{2}-s_{1}}=\frac{\varphi\left(s_{1}\right)-\varphi\left(s_{2}\right)}{s_{1}-s_{2}}
\end{aligned}
$$

which proves the inequality (3.9).

Now, if we choose

$$
t_{1}=y, t_{2}=\frac{x+y}{2}, s_{1}=\frac{y+z}{2} \text { and } s_{2}=\frac{x+z}{2}
$$

then we have $t_{1}<s_{1}$ and $t_{2}<s_{2}$ and by (3.9) we can write that

$$
\frac{\Phi(y)-\Phi\left(\frac{x+y}{2}\right)}{y-\frac{x+y}{2}} \leq \frac{\Phi\left(\frac{y+z}{2}\right)-\Phi\left(\frac{x+z}{2}\right)}{\frac{y+z}{2}-\frac{x+z}{2}}
$$

which is equivalent to the desired result (3.8).

(ii). We have from (i) that

$$
\mathscr{J}_{n, \Phi}(x, y)+\mathscr{g}_{n, \Phi}(y, z)+\mathscr{g}_{n, \Phi}(z, u) \leq \mathscr{g}_{n, \Phi}(x, u)
$$

and since $\mathscr{f}_{n, \Phi}(x, y), \mathscr{f}_{n, \Phi}(y, z) \geq 0$ we deduce the desired result (3.7).

Remark 2. With the assumptions of Theorem 4, we have the bound

$$
\sup _{x \leq y \leq z \leq u} \mathcal{I}_{n, \Phi}(y, z)=\mathcal{I}_{n, \Phi}(x, u) .
$$

For a constant $c \in \mathbb{R}$ we denote the vector having all components equal to this constant by $\bar{c}$, i.e., $\bar{c}=(c, \ldots, c) \in \mathbb{R}^{n}$. With this notation we have: $\mathbb{R}$.

Corollary 4. Let $\Phi: I \rightarrow \mathbb{R}$ be a convex function on the interval I of real numbers

(i) If $m, M \in I$ and $x \in I^{n}$ are such that $m \leq x_{i} \leq M$ for all $i \in\{1, \ldots, n\}$, then

$0 \leq \mathcal{J}_{n, \Phi}(\bar{m}, x)+\mathcal{J}_{n, \Phi}(x, \bar{M}) \leq n\left[\frac{1}{2}[\Phi(m)+\Phi(M)]-\Phi\left(\frac{m+M}{2}\right)\right]$

(ii) If $m, M \in I$ and $x, y \in I^{n}$ are such that $m \leq x_{i} \leq y_{i} \leq M$ for all $i \in$ $\{1, \ldots, n\}$, then

$$
0 \leq \mathcal{J}_{n, \Phi}(x, y) \leq n\left[\frac{1}{2}[\Phi(m)+\Phi(M)]-\Phi\left(\frac{m+M}{2}\right)\right] .
$$




\section{INEQUALITIES FOR DifFERENTIABLE FUnCTIONS}

The following result holds:

Theorem 5. Let $\Phi:[a, b] \rightarrow \mathbb{R}$ be a differentiable function on the interval $[a, b]$ of real numbers $\mathbb{R}$.

(i) If the derivative $\Phi^{\prime}$ is of bounded variation on $[a, b]$, then

$$
\begin{aligned}
\left|g_{n, \Phi}(x, y)\right| & \leq \frac{1}{4} \sum_{i=1}^{n}\left|y_{i}-x_{i}\right|\left|\bigvee_{x_{i}}^{y_{i}}\left(\Phi^{\prime}\right)\right| \leq \frac{1}{4} \bigvee_{a}^{b}\left(\Phi^{\prime}\right) \sum_{i=1}^{n}\left|y_{i}-x_{i}\right| \\
& =\frac{1}{2} \bigvee_{a}^{b}\left(\Phi^{\prime}\right) \delta(x, y)
\end{aligned}
$$

for any $x=\left(x_{1}, \ldots, x_{n}\right), y=\left(y_{1}, \ldots, y_{n}\right) \in[a, b]^{n}$.

The constant $\frac{1}{4}$ is best possible in both inequalities (4.1).

(ii) If the derivative $\Phi^{\prime}$ is $K$-Lipschitzian on $[a, b]$ with the constant $K>0$, then

$$
\left|\mathscr{g}_{n, \Phi}(x, y)\right| \leq \frac{1}{8} K \sum_{i=1}^{n}\left(y_{i}-x_{i}\right)^{2}=\frac{1}{2} K \mathcal{F}_{n, 2}(x, y)
$$

for any $x=\left(x_{1}, \ldots, x_{n}\right), y=\left(y_{1}, \ldots, y_{n}\right) \in[a, b]^{n}$.

The constant $\frac{1}{8}$ is best possible in (4.2).

Proof. For $x, y \in[a, b]$ with $x<y$ we consider the kernel $K:[x, y] \rightarrow \mathbb{R}$ defined by

$$
K_{x, y}(s):= \begin{cases}s-x & \text { if } x \leq s \leq \frac{x+y}{2} \\ y-s & \text { if } \frac{x+y}{2}<s \leq y .\end{cases}
$$

Integrating by parts in the Riemann-Stieltjes integral we have that

$$
\begin{aligned}
\int_{x}^{y} K_{x, y}(s) d\left[\Phi^{\prime}(s)\right] & =\int_{x}^{\frac{x+y}{2}}(s-x) d\left[\Phi^{\prime}(s)\right]+\int_{\frac{x+y}{2}}^{y}(y-s) d\left[\Phi^{\prime}(s)\right] \\
& =\left.(s-x) \Phi^{\prime}(s)\right|_{x} ^{\frac{x+y}{2}}-\int_{x}^{\frac{x+y}{2}} \Phi^{\prime}(s) d t \\
& +\left.(y-s) \Phi^{\prime}(s)\right|_{\frac{x+y}{2}} ^{y}+\int_{\frac{x+y}{2}}^{y} \Phi^{\prime}(s) d t \\
& =\frac{y-x}{2} \Phi^{\prime}\left(\frac{x+y}{2}\right)-\left[\Phi\left(\frac{x+y}{2}\right)-\Phi(x)\right] \\
& -\frac{y-x}{2} \Phi^{\prime}\left(\frac{x+y}{2}\right)+\left[\Phi(y)-\Phi\left(\frac{x+y}{2}\right)\right]
\end{aligned}
$$




$$
=2\left[\frac{\Phi(x)+\Phi(y)}{2}-\Phi\left(\frac{x+y}{2}\right)\right]
$$

i.e., we have the following representation of interest

$$
\frac{\Phi(x)+\Phi(y)}{2}-\Phi\left(\frac{x+y}{2}\right)=\frac{1}{2} \int_{x}^{y} K_{x, y}(s) d\left[\Phi^{\prime}(s)\right] .
$$

If $y<x$, then we also get

$$
\frac{\Phi(x)+\Phi(y)}{2}-\Phi\left(\frac{x+y}{2}\right)=\frac{1}{2} \int_{y}^{x} K_{y, x}(s) d\left[\Phi^{\prime}(s)\right] .
$$

(i) It is well known that, if $p:[\alpha, \beta] \rightarrow \mathbb{R}$ is continuous and $v:[\alpha, \beta] \rightarrow \mathbb{R}$ is of bounded variation, then the Riemann-Stieltjes integral $\int_{\alpha}^{\beta} p(s) d v(s)$ exists and

$$
\left|\int_{\alpha}^{\beta} p(s) d v(s)\right| \leq \sup _{s \in[\alpha, \beta]}|p(s)| \bigvee_{\alpha}^{\beta}(v) .
$$

If $x<y$, then on utilizing this property of the Riemann-Stieltjes integral we have successively

$$
\begin{aligned}
& \left|\frac{\Phi(x)+\Phi(y)}{2}-\Phi\left(\frac{x+y}{2}\right)\right| \\
& =\frac{1}{2}\left|\int_{x}^{y} K_{x, y}(s) d\left[\Phi^{\prime}(s)\right]\right| \\
& =\frac{1}{2}\left|\int_{x}^{\frac{x+y}{2}}(s-x) d\left[\Phi^{\prime}(s)\right]+\int_{\frac{x+y}{2}}^{y}(y-s) d\left[\Phi^{\prime}(s)\right]\right| \\
& \leq \frac{1}{2}\left|\int_{x}^{\frac{x+y}{2}}(s-x) d\left[\Phi^{\prime}(s)\right]\right|+\frac{1}{2}\left|\int_{\frac{x+y}{2}}^{y}(y-s) d\left[\Phi^{\prime}(s)\right]\right| \\
& \leq \frac{y-x}{4} \bigvee_{x}^{\frac{x+y}{2}}\left(\Phi^{\prime}\right)+\frac{y-x}{4} \bigvee_{\frac{x+y}{2}}^{y}\left(\Phi^{\prime}\right)=\frac{1}{4}(y-x) \bigvee_{x}^{y}\left(\Phi^{\prime}\right)
\end{aligned}
$$

and, similarly, if $y<x$, then

$$
\left|\frac{\Phi(x)+\Phi(y)}{2}-\Phi\left(\frac{x+y}{2}\right)\right| \leq \frac{1}{4}(x-y) \bigvee_{y}^{x}\left(\Phi^{\prime}\right) .
$$

Therefore, for any $x, y \in[a, b]$ we have the following inequality

$$
\left|\frac{\Phi(x)+\Phi(y)}{2}-\Phi\left(\frac{x+y}{2}\right)\right| \leq \frac{1}{4}|y-x|\left|\bigvee_{x}^{y}\left(\Phi^{\prime}\right)\right|
$$


which written for $x_{i}, y_{i} \in[a, b], i \in\{1, \ldots, n\}$ produces by summation the desired result (4.1).

In order to prove the sharpness of the constant $\frac{1}{4}$ in both inequalities from (4.1), assume that there exists a constant $G>0$ such that

$$
\left|\frac{\Phi(a)+\Phi(b)}{2}-\Phi\left(\frac{a+b}{2}\right)\right| \leq G(b-a) \bigvee_{a}^{b}\left(\Phi^{\prime}\right) \text {. }
$$

Consider the function $\Phi:[a, b] \rightarrow \mathbb{R}, \Phi(t)=\left|t-\frac{a+b}{2}\right|$. This function is absolutely continuous, $\Phi^{\prime}(t)=\operatorname{sgn}\left(t-\frac{a+b}{2}\right), t \in[a, b] \backslash\left\{\frac{a+b}{2}\right\}$ and $\bigvee_{a}^{b}\left(\Phi^{\prime}\right)=2$. Thus, (4.3) becomes

$$
\frac{b-a}{2} \leq 2 G(b-a),
$$

which implies that $G \geq \frac{1}{4}$.

(ii) We utilize the fact that for an $L$-Lipschitzian function, $p:[\alpha, \beta] \rightarrow \mathbb{R}$ and a Riemann integrable function $v:[\alpha, \beta] \rightarrow \mathbb{R}$, the Riemann-Stieltjes integral $\int_{\alpha}^{\beta} p(s) d v(s)$ exists and

$$
\left|\int_{\alpha}^{\beta} p(s) d v(s)\right| \leq L \int_{\alpha}^{\beta}|p(s)| d s .
$$

If $x<y$, then on utilizing this property of the Riemann-Stieltjes integral we have successively

$$
\begin{aligned}
& \left|\frac{\Phi(x)+\Phi(y)}{2}-\Phi\left(\frac{x+y}{2}\right)\right| \\
& =\frac{1}{2}\left|\int_{x}^{y} K_{x, y}(s) d\left[\Phi^{\prime}(s)\right]\right| \\
& =\frac{1}{2}\left|\int_{x}^{\frac{x+y}{2}}(s-x) d\left[\Phi^{\prime}(s)\right]+\int_{\frac{x+y}{2}}^{y}(y-s) d\left[\Phi^{\prime}(s)\right]\right| \\
& \leq \frac{1}{2}\left|\int_{x}^{\frac{x+y}{2}}(s-x) d\left[\Phi^{\prime}(s)\right]\right|+\frac{1}{2}\left|\int_{\frac{x+y}{2}}^{y}(y-s) d\left[\Phi^{\prime}(s)\right]\right| \\
& \leq \frac{1}{2} K\left[\int_{x}^{\frac{x+y}{2}}(s-x) d s+\int_{\frac{x+y}{2}}^{y}(y-s) d s\right]=\frac{1}{8} K(y-x)^{2} .
\end{aligned}
$$

The same inequality holds if $y<x$, and the desired inequality (4.2) is thus obtained.

To prove the sharpness of the constant $\frac{1}{8}$ in (4.2), let us assume that there exist $U>0$ so that

$$
\left|\frac{\Phi(a)+\Phi(b)}{2}-\Phi\left(\frac{a+b}{2}\right)\right| \leq U K(b-a)^{2} .
$$


Consider $\Phi:[a, b] \rightarrow \mathbb{R}, \Phi(t)=\frac{1}{2}\left(t-\frac{a+b}{2}\right)^{2}$. Then $\Phi^{\prime}(t)=t-\frac{a+b}{2}$ is Lipschitzian with the constant $K=1$ and (4.4) becomes

$$
\frac{1}{8}(b-a)^{2} \leq U(b-a)^{2},
$$

which implies that $U \geq \frac{1}{8}$.

Corollary 5. Let $\Phi:[a, b] \rightarrow \mathbb{R}$ be a differentiable function on the interval $[a, b]$ of real numbers $\mathbb{R}$. If the derivative $\Phi^{\prime}$ is $(\Delta, \delta)$-Lipschitzian on $[a, b]$ with the constant $\Delta>\delta$, then

$$
\begin{aligned}
\left|g_{n, \Phi}(x, y)-\frac{\Delta+\delta}{4} g_{n, 2}(x, y)\right| & \leq \frac{1}{16}(\Delta-\delta) \sum_{i=1}^{n}\left(y_{i}-x_{i}\right)^{2} \\
& =\frac{1}{4}(\Delta-\delta) g_{n, 2}(x, y),
\end{aligned}
$$

for any $x=\left(x_{1}, \ldots, x_{n}\right), y=\left(y_{1}, \ldots, y_{n}\right) \in[a, b]^{n}$. The constant $\frac{1}{16}$ is best possible in (4.5).

In particular, if $\Phi$ is twice differentiable and the second derivative $\Phi^{\prime \prime}$ satisfies the inequality $-\infty<\delta \leq \Phi^{\prime \prime}(t) \leq \Delta<\infty$ for each $t \in(a, b)$, then (4.5) is valid as well.

Proof. Follows by the statement (ii) from Theorem 5 on noticing that the function $\Phi-\frac{\Delta+\delta}{4} e^{2}$ has the property that $\Phi^{\prime}-\frac{\Delta+\delta}{2} e$ is $\frac{\Delta-\delta}{2}$-Lipschitzian on $[a, b]$. The details are omitted.

Theorem 6. Let $\Phi:[a, b] \rightarrow \mathbb{R}$ be a differentiable function on the interval $[a, b]$ of real numbers $\mathbb{R}$.

(i) If the derivative $\Phi^{\prime}$ is absolutely continuous on $[a, b]$, then

$$
\begin{aligned}
& \left|\mathcal{F}_{n, \Phi}(x, y)\right| \\
& \leq \begin{cases}\frac{1}{16} \sum_{i=1}^{n}\left(y_{i}-x_{i}\right)^{2} & \\
\times\left[\left\|\Phi^{\prime \prime}\right\|_{\left[x_{i}, \frac{x_{i}+y_{i}}{2}\right], \infty}+\left\|\Phi^{\prime \prime}\right\|_{\left[\frac{x_{i}+y_{i}}{2}, y_{i}\right], \infty}\right] & \text { if } \Phi^{\prime \prime} \in L_{\infty}[a, b] \\
\frac{1}{(q+1)^{1 / q} 2^{2+\frac{1}{q}}} \sum_{i=1}^{n}\left|y_{i}-x_{i}\right|^{1+\frac{1}{q}} & \text { if } \Phi^{\prime \prime} \in L_{p}[a, b], \\
\times\left[\left\|\Phi^{\prime \prime}\right\|_{\left[x_{i}, \frac{x_{i}+y_{i}}{2}\right], p}+\left\|\Phi^{\prime \prime}\right\|_{\left[\frac{x_{i}+y_{i}}{2}, y_{i}\right], p}\right] & p>1, \frac{1}{p}+\frac{1}{q}=1,\end{cases}
\end{aligned}
$$




$$
\leq \begin{cases}\frac{1}{8}\left\|\Phi^{\prime \prime}\right\|_{[a, b], \infty} \sum_{i=1}^{n}\left(y_{i}-x_{i}\right)^{2} & \text { if } \Phi^{\prime \prime} \in L_{\infty}[a, b] \\ \frac{1}{(q+1)^{1 / q} 2^{1+\frac{1}{q}}}\left\|\Phi^{\prime \prime}\right\|_{[a, b], p} \sum_{i=1}^{n}\left|y_{i}-x_{i}\right|^{1+\frac{1}{q}} & \text { if } \Phi^{\prime \prime} \in L_{p}[a, b], \\ p>1, \frac{1}{p}+\frac{1}{q}=1,\end{cases}
$$

for any $x=\left(x_{1}, \ldots, x_{n}\right), y=\left(y_{1}, \ldots, y_{n}\right) \in[a, b]^{n}$.

(ii) If the absolute value of the second derivative $\left|\Phi^{\prime \prime}\right|$ is convex on $[a, b]$, then

$$
\begin{aligned}
& \left|\mathcal{J}_{n, \Phi}(x, y)\right| \\
& \leq \frac{1}{12} \sum_{i=1}^{n}\left(y_{i}-x_{i}\right)^{2}\left[\frac{\left|\Phi^{\prime \prime}\left(y_{i}\right)\right|+\left|\Phi^{\prime \prime}\left(x_{i}\right)\right|}{4}+\left|\Phi^{\prime \prime}\left(\frac{x_{i}+y_{i}}{2}\right)\right|\right] \\
& \leq \frac{1}{16} \sum_{i=1}^{n}\left(y_{i}-x_{i}\right)^{2}\left[\left|\Phi^{\prime \prime}\left(y_{i}\right)\right|+\left|\Phi^{\prime \prime}\left(x_{i}\right)\right|\right] \\
& \leq \frac{1}{2}\left\|\Phi^{\prime \prime}\right\|_{[a, b], \infty} g_{n, 2}(x, y),
\end{aligned}
$$

$$
\text { for any } x=\left(x_{1}, \ldots, x_{n}\right), y=\left(y_{1}, \ldots, y_{n}\right) \in[a, b]^{n} \text {. }
$$

Proof. (i) Since $\Phi^{\prime}$ is absolutely continuous on $[a, b]$, then the Riemann-Stieltjes integral in the proof of Theorem 5 can be replaced with the Lebesgue integral and therefore we have

$$
\begin{aligned}
& \left|\frac{\Phi(x)+\Phi(y)}{2}-\Phi\left(\frac{x+y}{2}\right)\right| \\
& =\frac{1}{2}\left|\int_{x}^{y} K_{x, y}(s) \Phi^{\prime \prime}(s) d s\right| \\
& =\frac{1}{2}\left|\int_{x}^{\frac{x+y}{2}}(s-x) \Phi^{\prime \prime}(s) d s+\int_{\frac{x+y}{2}}^{y}(y-s) \Phi^{\prime \prime}(s) d s\right| \\
& \leq \frac{1}{2}\left|\int_{x}^{\frac{x+y}{2}}(s-x) \Phi^{\prime \prime}(s) d s\right|+\frac{1}{2}\left|\int_{\frac{x+y}{2}}^{y}(y-s) \Phi^{\prime \prime}(s) d s\right| \\
& \leq \frac{1}{2}\left[\int_{x}^{\frac{x+y}{2}}(s-x)\left|\Phi^{\prime \prime}(s)\right| d s+\int_{\frac{x+y}{2}}^{y}(y-s)\left|\Phi^{\prime \prime}(s)\right| d s\right]
\end{aligned}
$$

for any $x, y \in[a, b]$ with $x<y$.

Utilizing Hölder's integral inequality, we can state that

$$
\int_{x}^{\frac{x+y}{2}}(s-x)\left|\Phi^{\prime \prime}(s)\right| d s
$$




$$
\leq \begin{cases}\frac{(y-x)^{2}}{8}\left\|\Phi^{\prime \prime}\right\|_{\left[x, \frac{x+y}{2}\right], \infty} & \text { if } \Phi^{\prime \prime} \in L_{\infty}[a, b] \\ I(q)\left\|\Phi^{\prime \prime}\right\|_{\left[x, \frac{x+y}{2}\right], p} & \text { if } \Phi^{\prime \prime} \in L_{p}[a, b], p>1\end{cases}
$$

where

$$
I(q)=\left[\int_{x}^{\frac{x+y}{2}}(s-x)^{q} d s\right]^{1 / q}
$$

and $\frac{1}{q}+\frac{1}{p}=1, p>1$.

Observe that

$$
\begin{aligned}
I(q) & =\left[\left.\frac{(s-x)^{q+1}}{q+1}\right|_{x} ^{\frac{x+y}{2}}\right]^{1 / q}=\frac{1}{(q+1)^{1 / q}}\left(\frac{y-x}{2}\right)^{1+\frac{1}{q}} \\
& =\frac{1}{(q+1)^{1 / q} 2^{1+\frac{1}{q}}}(y-x)^{1+\frac{1}{q}} .
\end{aligned}
$$

In a similar manner we also have the inequality

$$
\begin{aligned}
& \int_{\frac{x+y}{2}}^{y}(y-s)\left|\Phi^{\prime \prime}(s)\right| d s \\
& \leq \begin{cases}\frac{(y-x)^{2}}{8}\left\|\Phi^{\prime \prime}\right\|_{\left[\frac{x+y}{2}, y\right], \infty} & \text { if } \Phi^{\prime \prime} \in L_{\infty}[a, b] \\
I(q)\left\|\Phi^{\prime \prime}\right\|_{\left[\frac{x+y}{2}, y\right], p} & \text { if } \Phi^{\prime \prime} \in L_{p}[a, b], p>1\end{cases}
\end{aligned}
$$

where $I(q)$ is given in (4.10).

Utilizing (4.8), (4.9) and (4.11) we can state that

$$
\begin{aligned}
& \left|\frac{\Phi(x)+\Phi(y)}{2}-\Phi\left(\frac{x+y}{2}\right)\right| \\
& \leq \begin{cases}\frac{(y-x)^{2}}{16}\left[\left\|\Phi^{\prime \prime}\right\|_{\left[x, \frac{x+y}{2}\right], \infty}+\left\|\Phi^{\prime \prime}\right\|_{\left.\left[\frac{x+y}{2}, y\right], \infty\right]}\right] & \text { if } \Phi^{\prime \prime} \in L_{\infty}[a, b] \\
\frac{1}{(q+1)^{1 / q} 2^{2+\frac{1}{q}}}|y-x|^{1+\frac{1}{q}} & \text { if } \Phi^{\prime \prime} \in L_{p}[a, b], \\
\times\left[\left\|\Phi^{\prime \prime}\right\|_{\left[x, \frac{x+y}{2}\right], p}+\left\|\Phi^{\prime \prime}\right\|_{\left.\left[\frac{x+y}{2}, y\right], p\right]}\right] & p>1, \frac{1}{p}+\frac{1}{q}=1,\end{cases}
\end{aligned}
$$

for any $x, y \in[a, b]$. 
(ii). It is well know that, if $\varphi:[\alpha, \beta] \rightarrow \mathbb{R}$ is a convex function, then for each $s \in[\alpha, \beta]$ we have the inequality

$$
\varphi(s) \leq \frac{(s-\alpha) \varphi(\beta)+(\beta-s) \varphi(\alpha)}{\beta-\alpha} .
$$

Utilizing this property and the fact that $\left|\Phi^{\prime \prime}\right|$ is convex, we have for $x<y$ that

$$
\left|\Phi^{\prime \prime}(s)\right| \leq \frac{(s-x)\left|\Phi^{\prime \prime}\left(\frac{x+y}{2}\right)\right|+\left(\frac{x+y}{2}-s\right)\left|\Phi^{\prime \prime}(x)\right|}{\frac{y-x}{2}}
$$

for any $s \in\left[x, \frac{x+y}{2}\right]$ and

$$
\left|\Phi^{\prime \prime}(s)\right| \leq \frac{\left(s-\frac{x+y}{2}\right)\left|\Phi^{\prime \prime}(y)\right|+(y-s)\left|\Phi^{\prime \prime}\left(\frac{x+y}{2}\right)\right|}{\frac{y-x}{2}}
$$

for any $s \in\left[\frac{x+y}{2}, y\right]$.

These imply the inequalities

$$
(s-x)\left|\Phi^{\prime \prime}(s)\right| \leq \frac{(s-x)^{2}\left|\Phi^{\prime \prime}\left(\frac{x+y}{2}\right)\right|+\left(\frac{x+y}{2}-s\right)(s-x)\left|\Phi^{\prime \prime}(x)\right|}{\frac{y-x}{2}}
$$

for any $s \in\left[x, \frac{x+y}{2}\right]$ and

$$
(y-s)\left|\Phi^{\prime \prime}(s)\right| \leq \frac{\left(s-\frac{x+y}{2}\right)(y-s)\left|\Phi^{\prime \prime}(y)\right|+(y-s)^{2}\left|\Phi^{\prime \prime}\left(\frac{x+y}{2}\right)\right|}{\frac{y-x}{2}}
$$

for any $s \in\left[\frac{x+y}{2}, y\right]$.

Integrating (4.13) on $\left[x, \frac{x+y}{2}\right]$ and (4.14) on $\left[\frac{x+y}{2}, y\right]$ we get

$$
\begin{aligned}
\int_{x}^{\frac{x+y}{2}}(s-x)\left|\Phi^{\prime \prime}(s)\right| d s & \leq \frac{2\left|\Phi^{\prime \prime}\left(\frac{x+y}{2}\right)\right|}{y-x} \int_{x}^{\frac{x+y}{2}}(s-x)^{2} d s \\
& +\frac{2\left|\Phi^{\prime \prime}(x)\right|}{y-x} \int_{x}^{\frac{x+y}{2}}\left(\frac{x+y}{2}-s\right)(s-x) d s
\end{aligned}
$$

and

$$
\begin{aligned}
\int_{\frac{x+y}{2}}^{y}(y-s)\left|\Phi^{\prime \prime}(s)\right| d s & \leq \frac{2\left|\Phi^{\prime \prime}(y)\right|}{y-x} \int_{\frac{x+y}{2}}^{y}\left(s-\frac{x+y}{2}\right)(y-s) d s \\
& +\frac{2\left|\Phi^{\prime \prime}\left(\frac{x+y}{2}\right)\right|}{y-x} \int_{\frac{x+y}{2}}^{y}(y-s)^{2} d s .
\end{aligned}
$$


Since simple calculations reveal that:

$$
\begin{aligned}
\int_{x}^{\frac{x+y}{2}}(s-x)^{2} d s & =\frac{(y-x)^{3}}{24}, \\
\int_{x}^{\frac{x+y}{2}}\left(\frac{x+y}{2}-s\right)(s-x) d s & =\frac{(y-x)^{3}}{48} \\
\int_{\frac{x+y}{2}}^{y}\left(s-\frac{x+y}{2}\right)(y-s) d s & =\frac{(y-x)^{3}}{48}, \\
\text { and } \int_{\frac{x+y}{2}}^{y}(y-s)^{2} d s & =\frac{(y-x)^{3}}{24},
\end{aligned}
$$

then (4.15) and (4.16) become

$$
\int_{x}^{\frac{x+y}{2}}(s-x)\left|\Phi^{\prime \prime}(s)\right| d s \leq \frac{(y-x)^{2}}{12}\left|\Phi^{\prime \prime}\left(\frac{x+y}{2}\right)\right|+\frac{(y-x)^{2}}{24}\left|\Phi^{\prime \prime}(x)\right|
$$

and

$$
\int_{\frac{x+y}{2}}^{y}(y-s)\left|\Phi^{\prime \prime}(s)\right| d s \leq \frac{(y-x)^{2}}{24}\left|\Phi^{\prime \prime}(y)\right|+\frac{(y-x)^{2}}{12}\left|\Phi^{\prime \prime}\left(\frac{x+y}{2}\right)\right| .
$$

Further on, by making use of the inequality (4.8) we deduce

$$
\begin{aligned}
& \left|\frac{\Phi(x)+\Phi(y)}{2}-\Phi\left(\frac{x+y}{2}\right)\right| \\
& \leq \frac{1}{2}\left[\int_{x}^{\frac{x+y}{2}}(s-x)\left|\Phi^{\prime \prime}(s)\right| d s+\int_{\frac{x+y}{2}}^{y}(y-s)\left|\Phi^{\prime \prime}(s)\right| d s\right] \\
& \leq \frac{1}{12}(y-x)^{2}\left[\frac{\left|\Phi^{\prime \prime}(y)\right|+\left|\Phi^{\prime \prime}(x)\right|}{4}+\left|\Phi^{\prime \prime}\left(\frac{x+y}{2}\right)\right|\right]
\end{aligned}
$$

for any $x, y \in[a, b]$.

The first inequality in (4.7) follows then easily from (4.17). The other two inequalities are obvious.

\section{APPLiCATIONS}

For the convex function $\Phi: I \subset(0, \infty) \rightarrow \mathbb{R}$ with $\Phi(t)=-\ln t$ we define the Jensen divergence

$$
\mathcal{F}_{n, 0}(x, y):=\sum_{i=1}^{n}\left\{\ln \left(\frac{x_{i}+y_{i}}{2}\right)-\frac{1}{2}\left[\ln \left(x_{i}\right)+\ln \left(y_{i}\right)\right]\right\},(x, y) \in I^{n} \times I^{n}
$$


which can be written as

$$
g_{n, 0}(x, y)=\ln \left[\prod_{i=1}^{n}\left(\frac{\frac{x_{i}+y_{i}}{2}}{\sqrt{x_{i} y_{i}}}\right)\right],(x, y) \in I^{n} \times I^{n} .
$$

If one wants to compare the Jensen divergence for a convex function $\Phi:[m, M] \rightarrow$ $\mathbb{R}$ with $\delta(x, y):=\frac{1}{2} \sum_{i=1}^{n}\left|x_{i}-y_{i}\right|$, the statistical distance between $x$ and $y$, then one has from (2.7) the following inequality

$$
0 \leq \mathcal{A}_{n, \Phi}(x, y) \leq \frac{1}{2}\left(\Phi_{-}^{\prime}(M)-\Phi_{+}^{\prime}(m)\right) \delta(x, y)
$$

for any $x, y \in[m, M]^{n}$, provided the lateral derivatives $\Phi_{-}^{\prime}(M)$ and $\Phi_{+}^{\prime}(m)$ are finite.

For instance, if we apply the inequality (5.1) to the function $\Phi(t)=-\ln t$ defined on the interval $[m, M] \subset(0, \infty)$, then we get

$$
0 \leq \mathcal{I}_{n, 0}(x, y) \leq \frac{M-m}{2 m M} \cdot \delta(x, y)
$$

for any $x, y \in[m, M]^{n}$.

The same inequality (5.1) applied for the convex function $\Phi(t)=t \ln t$ defined on the interval $[m, M] \subset(0, \infty)$ produces the result

$$
0 \leq \mathcal{g}_{n, 1}(x, y) \leq \ln \sqrt{\frac{M}{m}} \cdot \delta(x, y)
$$

for any $x, y \in[m, M]^{n}$.

As another example, we can consider the convex function $\Phi(t)=\exp t, t \in \mathbb{R}$. If we apply the inequality (5.1) to this function, we get

$$
0 \leq \mathcal{A}_{n, \exp }(x, y) \leq \frac{1}{2}(\exp (M)-\exp (m)) \delta(x, y)
$$

for any $x, y \in[m, M]^{n}$.

Now, if one wants to compare the Jensen divergence for a twice differentiable convex function $\Phi:[m, M] \rightarrow \mathbb{R}$ satisfying the condition $0 \leq \delta \leq \Phi^{\prime \prime}(t) \leq \Delta<\infty$ for any $t \in(m, M)$, with

$$
g_{n, 2}(x, y)=\sum_{i=1}^{n}\left[\frac{1}{2}\left(x_{i}^{2}+y_{i}^{2}\right)-\left(\frac{x_{i}+y_{i}}{2}\right)^{2}\right]=\frac{1}{4} \sum_{i=1}^{n}\left(x_{i}-y_{i}\right)^{2},
$$

that one has from (4.5) the following double inequality

$$
\frac{1}{2} \delta \mathscr{J}_{n, 2}(x, y) \leq \mathscr{J}_{n, \Phi}(x, y) \leq \frac{1}{2} \Delta \mathscr{J}_{n, 2}(x, y),
$$

for any $x, y \in[m, M]^{n}$. 
If we apply the inequality (5.5) to the function $\Phi(t)=-\ln t$ defined on the interval $[m, M] \subset(0, \infty)$, then we get

$$
\frac{1}{2 M^{2}} \mathcal{g}_{n, 2}(x, y) \leq \mathcal{A}_{n, 0}(x, y) \leq \frac{1}{2 m^{2}} \mathcal{g}_{n, 2}(x, y)
$$

for any $x, y \in[m, M]^{n}$.

The same inequality (5.5) applied to the convex function $\Phi(t)=t \ln t$ defined on the interval $[m, M] \subset(0, \infty)$ produces the result

$$
\frac{1}{2 M} g_{n, 2}(x, y) \leq \mathcal{A}_{n, 1}(x, y) \leq \frac{1}{2 m} g_{n, 2}(x, y),
$$

for any $x, y \in[m, M]^{n}$.

Finally, if we apply the inequality (5.5) to the convex function $\Phi(t)=\exp t$ on the interval $[m, M] \subset \mathbb{R}$, then we get the bounds

$$
\frac{1}{2} g_{n, 2}(x, y) \exp m \leq g_{n, \exp }(x, y) \leq \frac{1}{2} g_{n, 2}(x, y) \exp M,
$$

for any $x, y \in[m, M]^{n}$.

\section{REFERENCES}

[1] J. Aczél and Z. Daróczy, On measures of information and their characterizations. New York: Academic Press [Harcourt Brace Jovanovich Publishers], 1975, mathematics in Science and Engineering, Vol. 115.

[2] J. Burbea and C. R. Rao, "On the convexity of some divergence measures based on entropy functions," IEEE Trans. Inform. Theory, vol. 28, no. 3, pp. 489-495, 1982. [Online]. Available: http://dx.doi.org/10.1109/TIT.1982.1056497

[3] R. G. Gallager, Information Theory and Reliable Communication. New York: Wiley, 1968.

[4] J. Havrda and F. Charvát, "Quantification method of classification processes. Concept of structural a-entropy," Kybernetika (Prague), vol. 3, pp. 30-35, 1967.

[5] N. Jardine and R. Sibson, Mathematical taxonomy. London: John Wiley \& Sons Ltd., 1971, wiley Series in Probability and Mathematical Statistics.

[6] R. C. Lewonton, “The apportionment of human diversity,” Evolutionary Biology, vol. 6, pp. 381398, 1972.

[7] Z. Liu, "Refinement of an inequality of Grüss type for Riemann-Stieltjes integral," Soochow J. Math., vol. 30, no. 4, pp. 483-489, 2004.

[8] M. L. Menéndez, J. A. Pardo, L. Pardo, and M. C. Pardo, "The Jensen-Shannon divergence," J. Franklin Inst. B, vol. 334, no. 2, pp. 307-318, 1997. [Online]. Available: http://dx.doi.org/10.1016/S0016-0032(96)00063-4

[9] L. Pardo, D. Morales, and I. J. Taneja, "Generalized Jensen difference divergence measures and Fisher measure of information," Kybernetes, vol. 24, no. 2, pp. 2, 15-28, 1995. [Online]. Available: http://dx.doi.org/10.1108/03684929510083066

[10] E. C. Pielou, Ecological Diversity. New York: Wiley, 1975.

[11] A. P. Sant'Anna and I. J. Taneja, "Trigonometric entropies, Jensen difference divergence measures, and error bounds," Inform. Sci., vol. 35, no. 2, pp. 145-156, 1985. [Online]. Available: http://dx.doi.org/10.1016/0020-0255(85)90046-5

[12] R. Sibson, "Information radius," Z. Wahrscheinlichkeitstheorie und Verw. Gebiete, vol. 14, pp. 149-160, 1969/1970. 
[13] S.-C. Tsai, W.-G. Tzeng, and H.-L. Wu, "On the Jensen-Shannon divergence and variational distance," IEEE Trans. Inform. Theory, vol. 51, no. 9, pp. 3333-3336, 2005. [Online]. Available: http://dx.doi.org/10.1109/TIT.2005.853308

Authors' addresses

\section{S. S. Dragomir}

School of Computational \& Applied Mathematics, University of the Witwatersrand, Private Bag-3, Wits-2050, Johannesburg, South Africa

Current address: Mathematics, School of Engineering \& Science, Victoria University, PO Box 14428, Melbourne City, MC 8001, Australia

E-mail address: Sever.Dragomir@vu.edu.au

\section{N. M. Dragomir}

School of Biomedical \& Health Sciences, Victoria University PO Box 14428, Melbourne City, MC 8001, Australia

E-mail address: Nicoleta.Dragomir@vu.edu.au

\section{Sherwell}

School of Computational \& Applied Mathematics, University of the Witwatersrand, Private Bag-3, Wits-2050, Johannesburg, South Africa

E-mail address: David. Sherwell@wits.ac.za 